\title{
VALORES DE IRISINA E SUA CORRELAÇAO COM A COMPOSIÇAO CORPORAL EM CRIANÇAS COM ADRENARCA PRECOCE
}

\section{Pôster}

Autores deste trabalho:

Vanessa de Oliveira Furino: Departamento de Enfermagem- Universidade Federal de São Carlos (UFSCar)

Fernanda de Oliveira Furino : Departamento de Enfermagem- Universidade Federal de São Carlos (UFSCar)

Lucimar Retto da Silva de Avó: Departamento de Medicina -Universidade Federal de São Carlos (UFSCar)

Debora Gusmão Melo: Departamento de Medicina -Universidade Federal de São Carlos (UFSCar)

Lucila Leico Kagohara Elias: Departamento de Fisiologia -Universidade de São Paulo (USP)

Carla Maria Ramos Germano : Departamento de Medicina -Universidade Federal de São Carlos (UFSCar)

Área do Trabalho: Pediatria

Número de inscrição: 5247

Data da submissão:05/08/2016 às 17:05

\section{Justificativa}

A adrenarca precoce (AP) é caracterizada pelo surgimento precoce de pelos pubianos e/ou axilares, acne e odor corporal e estes indivíduos têm risco de desenvolver síndrome metabólica, DM2 e SOP. A irisina parece ter um papel ativo no metabolismo de carboidratos e lipídeos, no entanto, pouco se sabe do papel desse hormônio na AP.

\section{Objetivo(s)}

Analisar os valores de irisina em crianças com diagnóstico de AP e a relação desse hormônio com a composição corporal desses indivíduos.

\section{Método(s)}

Estudo quantitativo transversal exploratório, com 15 crianças com diagnóstico de AP e 15 controles (C) pareados, idade de 100,1 \pm 5,5 e 95,1 \pm 5,1 meses, respectivamente, dos 
quais $40 \%$ eram meninos $(n=12)$ e $60 \%$ eram meninas $(n=18)$. Os participantes foram recrutados na rede pública de saúde de São Carlos, SP e o estudo foi aprovado pelo CEPUFSCar. Foram determinados dados antropométricos: altura, peso, circunferência abdominal e prega cutânea tricipital. Os participantes realizaram exames de glicemia, insulina, DHEA-S, 17OHP e irisina. A análise dos dados foi realizada através do programa GraphPad Prism5. O teste de Wilcoxon pareado foi utilizado para avaliar a diferença entre as médias e sua correlação através do teste de Spearman, com nível de significância de $5 \%$.

\section{Resultado(s)}

Não houve diferença significativa em relação aos valores de irisina $(16,1 \pm 1,3 \mathrm{mg} / \mathrm{dl}$ nos indivíduos $\mathrm{C}$ versus $17,3 \pm 1,7$ nos indivíduos com $\mathrm{AP} ; \mathrm{p}=0,5880$ ) entre os grupos. A categorização dos grupos em relação à circunferência abdominal (CA) evidenciou que 6,7\% do grupo $\mathrm{C}$ versus 33,3\% do grupo AP tinha CA acima do p90. Adicionalmente, $6,7 \%$ do grupo $\mathrm{C}$ versus $20 \%$ do grupo AP apresentavam relação $\mathrm{G} / \mathrm{I}<7$ e insulina $>$ 15\&\#956;UI/ml. Nos grupos C e AP, a correlação da relação glicemia/insulina com a irisina foi, respectivamente, de $-0,5915(\mathrm{p}=0,07)$ e $-0,7033(\mathrm{p}=0,007)$.

\section{Conclusão(ões)}

Embora este estudo não tenha evidenciado diferenças significativas nos valores de irisina entre os grupos C e AP, foi evidenciada maior proporção de indivíduos com hiperinsulinemia, menor relação G/I e CA elevada em crianças do grupo AP. Estes parâmetros são importantes marcadores de SM, DM2 e DCV futuras. Essas crianças, portanto, devem ter um acompanhamento clínico adequado e de longo prazo. 